\title{
Mosaic trisomy 14
}

INSERM

\section{Source}

INSERM. (1999). Orphanet: an online rare disease and orphan drug data base. Mosaic trisomy 14. ORPHA:1703

Mosaic trisomy 14 is a rare chromosomal anomaly disorder, with a highly variable phenotype, principally characterized by growth and developmental delay, intellectual disability, body asymmetry/hypotonia, congenital heart defects, genitourinary abnormalities (cryptorchidism, micropenis, large clitoris, labial swelling), and abnormal skin hyperpigmentation. Patients usually present with craniofacial dysmorphism such as microcephaly, abnormal palpebral fissure, hypertelorism, ear abnormalities, broad nose, low-set ears, micro/retro-gnathia, and cleft or highly arched palate. 